\title{
Melting-layer modelling at C-Band
}

\author{
J. Steinert and M. Chandra \\ Professorship of Microwave Engineering and Photonics, Chemnitz University of Technology, Germany
}

\begin{abstract}
Stratiform cloud formations, such as a uniform type of precipitating clouds, are sub dividable into layers with different aggregate states. In this way, it is adaptable to measurements with polarimetric radar. An important task here is the detection of the transition zone between the liquid particles (raindrops) below and the frozen particles (ice crystals) above, i.e. the melting-layer. Linked to the physical behaviour of melting, the measured signatures, from this area, include ice crystals and raindrops in different aggregate stages. A given melting-layer model will be analysed and the comparison to models for raindrops and ice particles from earlier studies will be done. Then the results are compared with measured signatures from the C-Band radar, POLDIRAD (Oberpfaffenhofen). So we shall seek an answer to the question whether the results are sufficient to create a melting-layer-class for hydrometeor classification.
\end{abstract}

\section{Introduction}

By looking at raindrops it is usually not easy to understand how these formations results from frozen particles like ice or snow crystals. In the literature the treatise on raindrops is widely addressed (e.g. Steinert, 2008) and therewith it is curious why melting particles are out of scope. The difficulty with these particles is the change in shape and in the dielectric property. Nevertheless there are scattering models that fits the melting process well and build the base for this study. Not to start from the beginning, this contribution is focused on a short description of the measurement hardware and the explanation of the available polarimetric parameters in Sect. 2. In Sect. 3 we will speak about the signatures of the particles in the melting layer. Additionally the scatter-

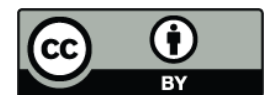

Correspondence to: J. Steinert (joerg.steinert@etit.tu-chemnitz.de) ing model and the combination of the simulation results with the measured values are given in Sects. 4 and 5. In Sect. 6 the possibility of a discrimination between melting and non melting particles will be addressed.

\section{Hardware and polarimetric parameter}

To analyse the weather events, recorded datasets from the pulsed dual polarimetric weather radar POLDIRAD (radar site Oberpfaffenhofen (Germany)) were taken. Technical details of the radar can be found in Schroth et al. (1988). The selected pulse width of $1 \mu$ s results in a range resolution of $150 \mathrm{~m}$ and specify with the beam width of $1^{\circ}$ the size of the pulse volume. Furthermore the complete four element scattering matrix was measured for every pulse volume. So that, in the polarization base linear horizontal/linear vertical, the four reflectivities $Z_{\mathrm{hh}}, Z_{\mathrm{vh}}, Z_{\mathrm{hv}}$ and $Z_{\mathrm{vv}}$ in dBZ are available from the measured power. From the two copolar reflectivities the differential reflectivity is estimated in $\mathrm{dB}$ with $Z_{\mathrm{DR}}=Z_{\mathrm{hh}}-Z_{\mathrm{vv}}$ and gives information about the mean shape of the particles in the pulse volume. $Z_{\mathrm{hh}}$ and $Z_{\mathrm{DR}}$ are the parameters of choice for the analysis of the data. To dissect the structure of the clouds, vertical cuts, the so called range height indicators (RHI), were taken as measurement geometry.

\section{Melting layer signatures}

Based on the adiabatic curve, the temperature changes (decreases) with height in the troposphere. Usually the melting as the transition between liquid and frozen hydrometeors start if the temperature oversteps $0{ }^{\circ} \mathrm{C}$. The associated height then corresponds to the $0^{\circ} \mathrm{C}$ isotherm. Additionally, this height is the upper limit of the so called melting layer. The lower limit is reached if all hydrometeors are completely

Published by Copernicus Publications on behalf of the URSI Landesausschuss in der Bundesrepublik Deutschland e.V. 


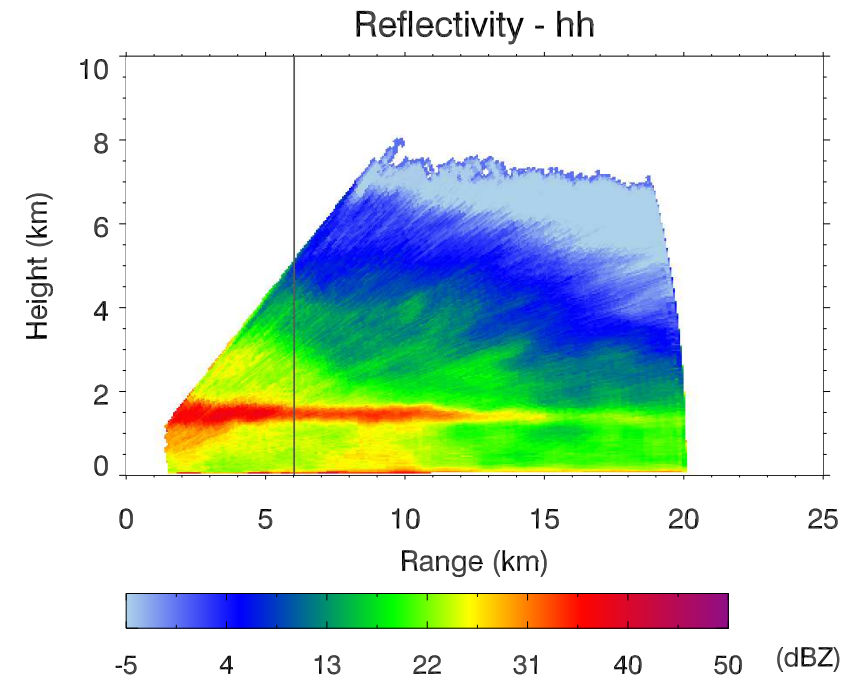

Fig. 1. RHI scan of the horizontal reflectivity of a stratiform dataset recorded on 16 November 1995 and the position of the extracted vertical profile at a distance of $6 \mathrm{~km}$ (dark grey line).

melted to raindrops. The vertical dimension of the melting layer range from several hundred meters up to $1 \mathrm{~km}$ or slightly more. Turbulences in the troposphere, like upwinds in convective cloud formations, disturb the creation of a melting layer and so it is well distinct just in calm stratiform events. From the RHI scans the melting layer is characterized by a horizontal band with higher reflectivities bounded by lower values below and above. So vertical profiles of the reflectivity were extracted from the scans to investigate the change in reflectivity and with it the bottom and the top limit of the melting zone. The estimation of the vertical reflectivity profiles from RHI datasets is described in detail in Steinert (2009). A record of a stratiform event is presented with $Z_{\mathrm{hh}}$ in Fig. 1. A vertical profile was for instance estimated at $6 \mathrm{~km}$ distance (grey line in Fig. 1) and is shown in Fig. 2 for $Z_{\mathrm{hh}}$ and $Z_{\mathrm{DR}}$. The heights of the maximum values of the two reflectivities (green dashed lines in Fig. 2) have a height difference in the area of $200 \mathrm{~m}$ due to the melting process (Dissanayake et al., 1983).

\section{Model description}

The simulation of the melting layer signatures was done by the model of Zhang (1996) whereas the basics are given in Zhang (1994). With this method the vertical height profiles in the melting region were estimated for the copolar reflectivities and the differential reflectivity. The assumption made in the model is that one crystal results in one raindrop so the coalescence of particles is not included. The simulation of $Z_{x y}$ follows the common procedure for the estimation of the reflectivity and can be expressed by Eq. (1).
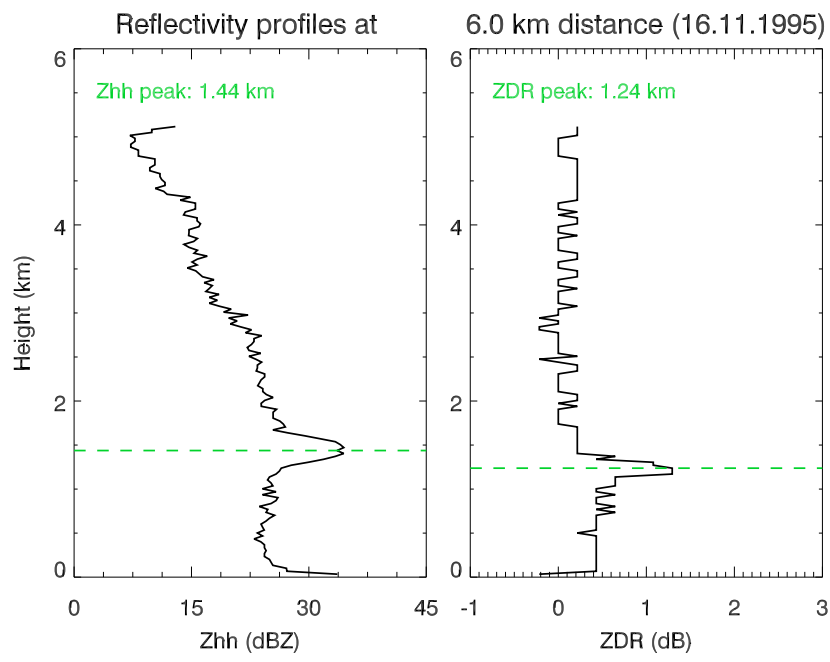

Fig. 2. Vertical profiles of $Z_{\mathrm{hh}}$ and $Z_{\mathrm{DR}}$ of the dataset displayed in Fig. 1 with the position of the maximum value of the desired parameter (dashed green line).

$Z_{x y}=10 \cdot \lg \left(\frac{\lambda^{4}}{\pi^{5} \cdot|K|^{2}} \cdot \int_{D_{e}} \sigma_{x y}\left(D_{e}\right) \cdot N\left(D_{e}\right) d D_{e}\right)$

The index $x$ is related to the polarization in receiving channel and $y$ corresponds to the polarization in transmitting direction. In the order of appearance the parameters are the wavelength of the radar $\lambda$ in $\mathrm{mm}$, the dielectric factor $K=\frac{\varepsilon-1}{\varepsilon+2}$ with the complex permittivity $\varepsilon$ of the particle. The integration of the radar cross section $\sigma_{x y}$ in $\mathrm{mm}^{2}$ and the particle size distribution $N\left(D_{e}\right)$ in $\frac{1}{\mathrm{~mm} \cdot \mathrm{m}^{3}}$ is done over the particle diameter $D_{e}$ in mm. Starting with the calculation of $\sigma_{\mathrm{hh}, \mathrm{vv}}$ of a single particle, the size, the shape and the dielectric constant have to be known. For the shape, a rotational spheroid was assumed with a changing axial ratio to size relation. This shape is equal to that of raindrops which corresponds to the similar electric behaviour due to the high dielectric constant of water in contrast to that of snow. The relation between the axial ratio AR (vertical half axis divided by the horizontal half axis of the spheroid) and the equivolume diameter $D_{e}$ (diameter of a sphere with the same volume as the spheroid) is $\mathrm{AR}=1-0.5 \cdot D_{e}$ (Zhang, 1996). The shape of the particles is needed for the estimation of $Z_{\mathrm{DR}}$ because the spheroidal shape give different backscattered powers for horizontal and vertical polarization. A key aspect in the model is, that the size of the resulted raindrop (radius $a_{2}$ ) can be directly estimated from the size of the frozen particle (radius $a$ ) with

$a_{2}^{3}=a^{3}\left[Q+(1+Q) \frac{\varrho_{\mathrm{w}}}{\varrho_{\mathrm{s}}}\right]($ Zhang, 1994)

with the densities $\varrho_{\mathrm{s}}$ for dry snow and $\varrho_{\mathrm{w}}$ for liquid water. $Q$ is the mass fraction of the mass of the melted water in the snow crystal. The size of the snow particles ranges from 


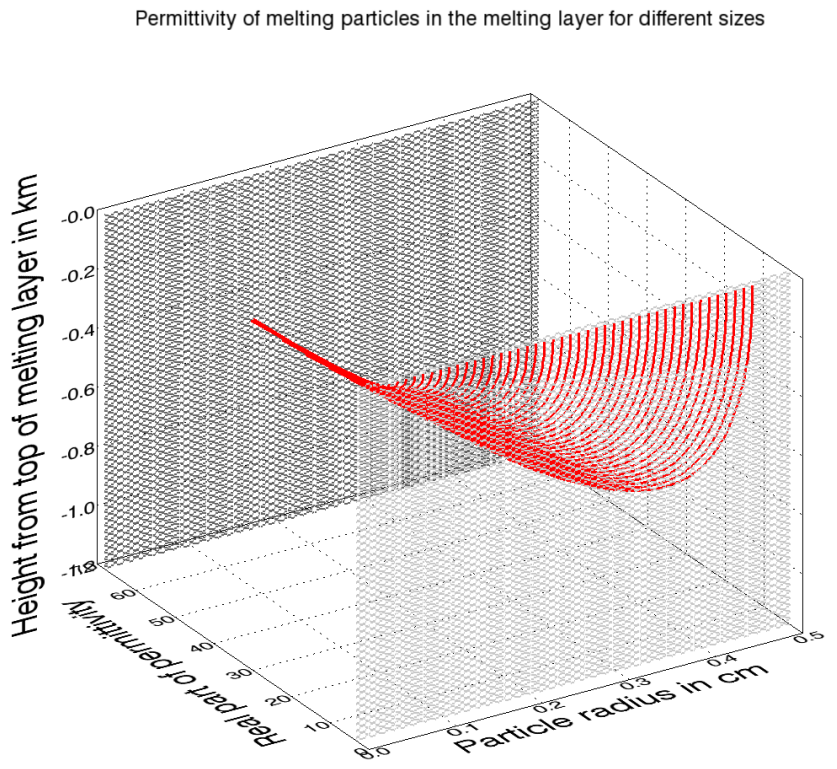

Fig. 3. Real part of the permittivity of the particles through melting over height for different sizes with real part of permittivity of water with a constant value of 68.21 (dark grey wall) and real part of permittivity of ice with a constant value of 3.17 (light grey wall).

radii $a$ between $0.025 \mathrm{~cm}$ and $0.325 \mathrm{~cm}$. So the resulted maximum radius of the raindrop will be $0.45 \mathrm{~cm}$. The dielectric constant depends on the stage within the melting process. Starting from ice, the variation of $\varepsilon$ to that of water relates to the height in the melting layer. So with a varying distance to the top of the melting layer, the inner structure changes from the frozen to the liquid state. The time or fall-height until the transformation is finished depends on the particle size, cf. Fig. 3.

So small particles need less time to melt as larger ones. The different particle sizes are connected to a gamma particle size distribution (Ulbrich, 1983). The coefficients $N_{0}$ and $\lambda$ are given in Zhang (1994) depending on the rainrate $(\mathrm{RR}$ in $\mathrm{mm} / \mathrm{h})$ with $N_{0}=1.42 \times 10^{10} \frac{1}{\mathrm{~cm}^{-4} \mathrm{~m}^{3}}$ and $\Lambda=$ $1.3 \cdot R R^{-0.13} \times 10^{2}$ and is associated to the relation $N(a)=$ $N_{0} \cdot a^{3} \cdot e^{-\Lambda a}$ with the radius $a$. Hence the parameter $\mu=3$ by using the equations from Ulbrich (1983).

The simulation result using these default parameters is shown in the next section in combination with a dataestimated height profile.

\section{Comparison of simulation and measurement}

The method started with the estimation of the rainrate from the reflectivity below the melting layer with the well known equation $\zeta=200 \cdot R R^{1.6}$ (Marshall and Palmer, 1948) with $Z=10 \cdot \log (\zeta)$. Then $N_{0}$, $\mu$ and the snow density $\varrho_{\mathrm{s}}$ were varied to reduce the mean squared error (MSE) between measured and
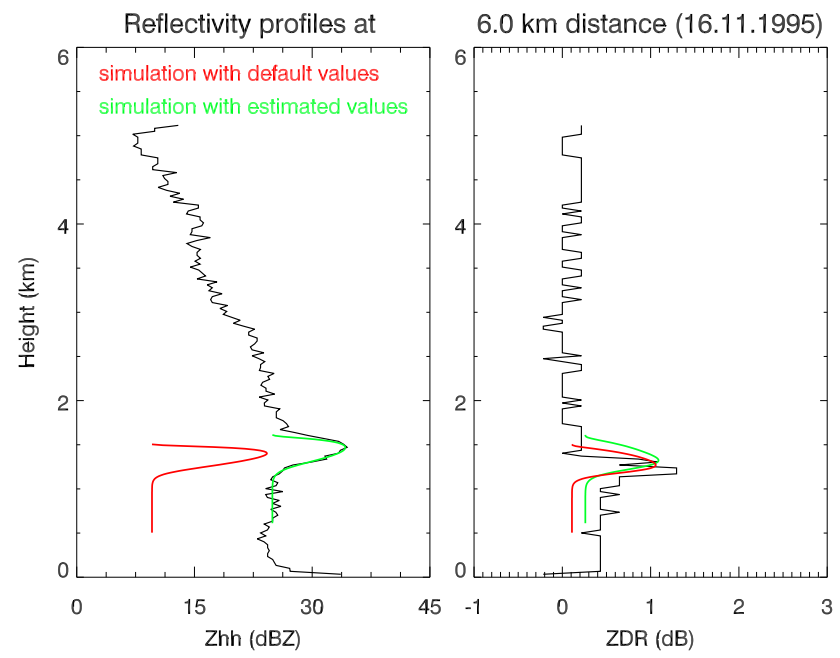

Fig. 4. Measured profiles of $Z_{\mathrm{hh}}$ and $Z_{\mathrm{DR}}$ (see also Fig. 2) and simulated profiles with default parameters (red curve) (Zhang, 1994) and with investigated parameters (green curve).

simulated reflectivities. The MSE is calculated with $\operatorname{MSE}=\left(\operatorname{bias}\left(f_{0}(x)-f_{1}(x)\right)\right)^{2}+\operatorname{var}\left(f_{0}(x)-f_{1}(x)\right)$ and is from the given parameters $\operatorname{MSE}\left(Z_{\mathrm{hh}}\right)_{\text {default }}=208.31$ and $\operatorname{MSE}\left(Z_{\mathrm{DR}}\right)_{\text {default }}=0.20$. As the differential reflectivity is in principle much lower than the copolar reflectivity, the MSE follows this behaviour. The best agreement resulted from the shape value with $\mu_{\text {estimated }}=3.43$ for the particle size distribution. Furthermore a higher count of particles with an additional factor of $N_{0, \text { factor }}=9.07$ altered the particle size density to Eq. (3),

$N\left(D_{e}\right)=3.18 \times 10^{10} \cdot D_{e}^{3.43} \cdot e^{-\frac{89.64 \cdot D_{e}}{R R^{0.12}}}$

An increase of the snow density by a multiplication with $\varrho_{\mathrm{s}, \text { factor }}=1.93$ leads beyond the size distribution to the best fit of the compared signatures. With these changes, MSE reduced to $\operatorname{MSE}\left(Z_{\mathrm{hh}}\right)_{\text {estimated }}=3.23$. For $Z_{\mathrm{DR}}$ the axial ratio of the spheroid is accountable to the fit. Therefore the selection of a different relation with

$\mathrm{AR}=1 .-D_{e}^{1.37}$.

decrease the MSE to $\operatorname{MSE}\left(Z_{\mathrm{DR}}\right)_{\text {estimated }}=0.15$. The obvious difference in height between the measured values and the simulated profiles are possibly in connection with a canting of the particles, something that was not accounted in this study.

The simulated profiles and the estimated ones are drawn together in Fig. 4 for the reflectivity and the differential reflectivity.

\section{Classification}

Liquid and frozen hydrometeors can be well separated in the $Z_{\mathrm{hh}}-Z_{\mathrm{DR}}$-plane (Steinert and Chandra, 2007). Nevertheless 


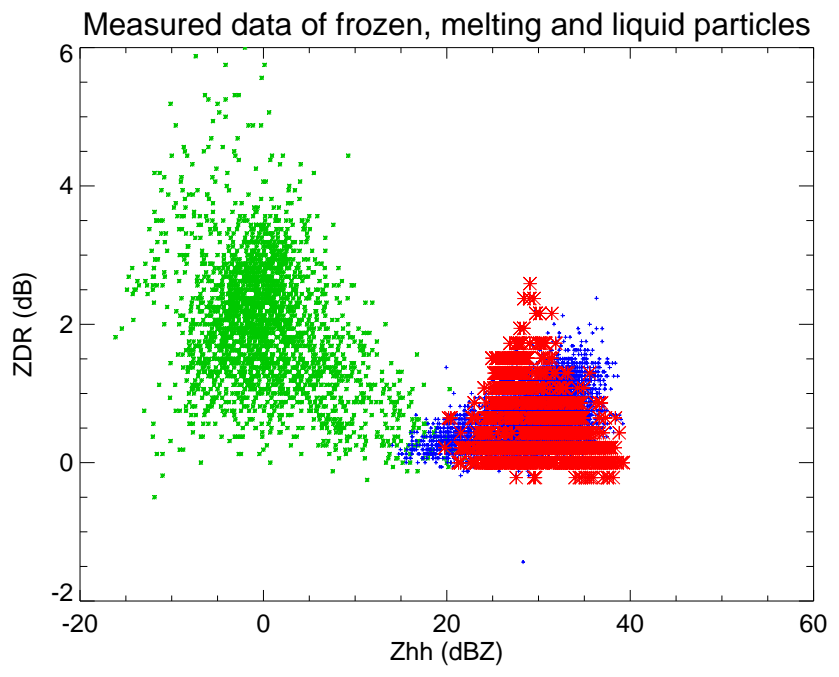

Fig. 5. $Z_{\mathrm{hh}}-Z_{\mathrm{DR}}$-plane with the measured signatures of ice crystals (green), raindrops (blue) and melting (red) particles selected by a manual inspection of several POLDIRAD measurements.

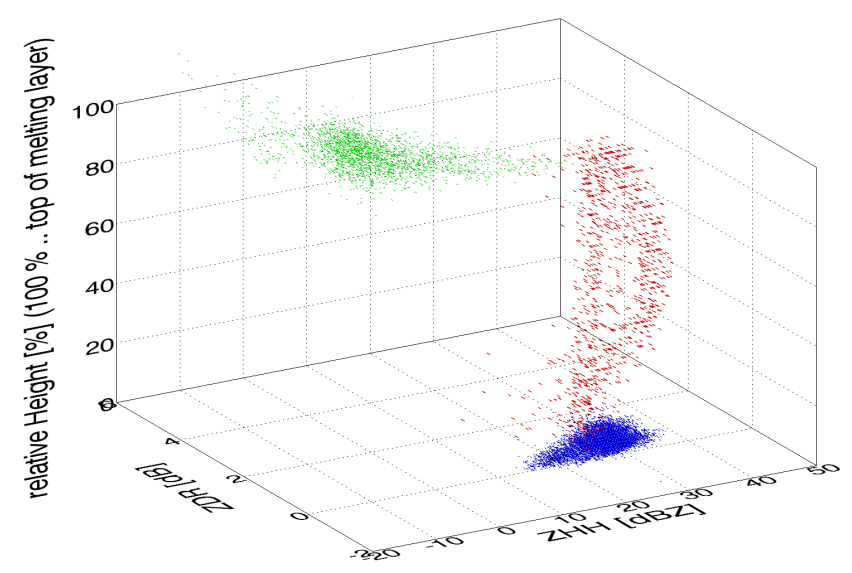

Fig. 6. Content of Fig. 5 but now in a 3-D representation with the height in relation to the top of the melting layer. Furthermore the shown signatures of the particles in melting layer (red color) are in contrast to the previous figure simulated and not real measured ones due to not known height of the melting layer.

the $Z_{\mathrm{DR}}$ values are in the same region for crystals and raindrops but the reflectivity values are different (cf., Fig. 5).

The difficulty is the coexistence of the signatures of melting and raindrop particles as also to be seen in Fig. 5. So a hydrometeor classification based on $Z_{\mathrm{hh}}$ and $Z_{\mathrm{DR}}$ alone is not possible. A solution will be the inclusion of the height in relation to the melting layer, because pulse volumes of pure liquid particles will be below and regions of frozen particles will be above the melting zone. The sketch in Fig. 6 give you an impression of the distribution of the signatures in relation to the melting layer height. For simplicity reasons the frozen particles are concentrated at the top and the raindrops are drawn at the bottom of the melting layer.
The consequence is, that for the classification the estimation of melting layer height is essential.

\section{Conclusions}

A short introduction in the backscattering simulation of particles in the melting layer was presented. Besides the correct shape of the particles the simulation shows, that the dielectric property and therewidth the dielectric constant is much more important for a good estimation of the signatures. An important result is that the choosen model from Zhang (1996) give with the default values reasonable results. Anyway the coefficients in the model were adapted for an improvement of the simulated reflectivities. As criteria for the comparison the MSE was picked out. Last but not least the agenda contained the classification of the hydrometeors. Here, similarities of the liquid and melting particles plays a role that can be resolved by the look on the height in the cloud.

\section{References}

Dissanayake, A. W., Chandra, M., and Watson, P. A.: Prediction of differential reflectivity due to various types of ice particles and ice-water mixtures, in: ICAP 83, Norwich, UK, pp. 56-59, IEE Publication No. 219, 1983.

Marshall, J. and Palmer, W.: The distribution of raindrops with size, J. Meteorol., 5, 165-166, 1948.

Schroth, A. C., Chandra, M. S., and Meischner, P. F.: A C-Band Coherent Polarimetric Radar for Propagation and Cloud Physics Research, J. Atmos. Oceanic Technol., 5, 803-822, 1988.

Steinert, J. and Chandra, M.: Reflectivity relationships of polarimetric C-band measurements of rain signatures, Proceedings of WFMN07, 30-34, http://archiv.tu-chemnitz.de/pub/2007/0210/, 2007.

Steinert, J. and Chandra, M.: Cloud physical properties and empirical polarimetric measurements of rain signatures at C-Band, Adv. Radio Sci., 6, 315-318, doi:10.5194/ars-6-315-2008, 2008.

Steinert, J. and Chandra, M.: Cloud profiles from C-band groundbased radar, in Proceedings of the 8th International Symposium on Tropospheric Profiling, 19-23 October 2009, Delft, The Netherlands, 2009.

Ulbrich, C. W.: Natural variations in analytical form of the raindrop size distribution, J. Climate Appl. Meteor., 22, 1764-1775, 1983.

Zhang, W.: Scattering of Radiowaves by a Melting Layer of Precipitation in Backward and Forward Directions, IEEE Trans. Antennas Propag., 42, 347-356, 1994.

Zhang, W., Tervonen, J. K., and Salonen, E. T.: Backward and Forward Scattering by the Melting Layer Composed of Spheroidal Hydrometeors at 5-100 GHz, IEEE Trans. Antennas Propag., 44, 1208-1219, 1996. 